\title{
Daria Hazuda
}

Merck \& Co. set up its Exploratory Science Center in Cambridge, Massachusetts, to keep a finger on the pulse of emerging biological science, with a disease-agnostic scope and nimble mindset. Headed up by Daria Hazuda, CSO of the centre and vice president of infectious diseases and vaccine discovery at Merck, for now it focuses on the interaction between the microbiome and human immunity. Hazuda spoke with Asher Mullard about the firm's exploratory science agenda, its interest in the microbiome and its continued focus on antibiotics and HIV drugs.

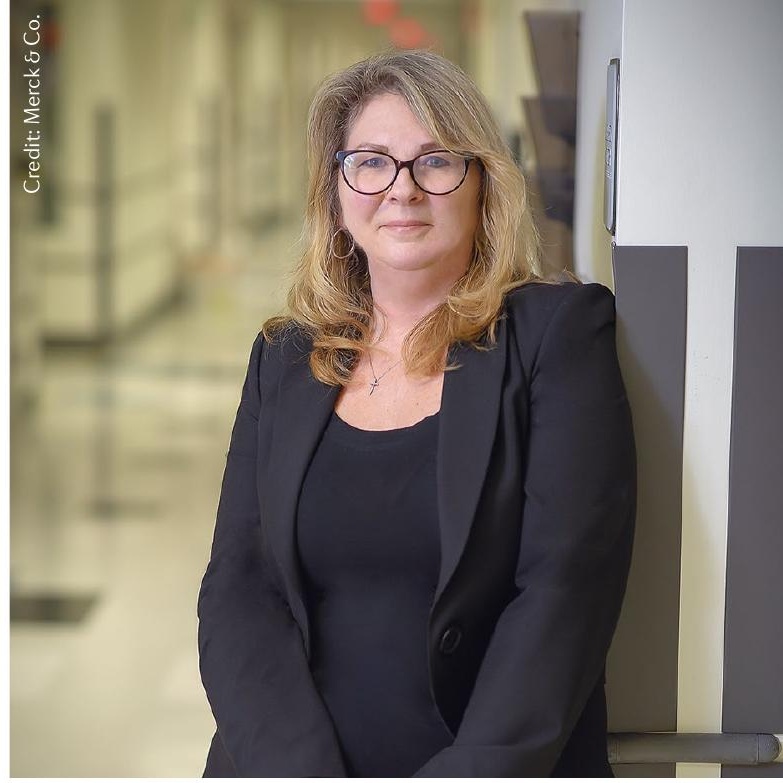

\section{Q What was Merck \& Co.s aim with the Exploratory Science Center in Cambridge? It's been a little over 2 years since we occupied space here, and we now have about 40 to 50 people working here at steady state. We are a group of interdisciplinary scientists - biologists, data scientists, chemists and pharmacologists - who can focus on emerging new areas of biology that are still early-stage but look like they have long-term potential to offer major impacts on human health. We are disease area-agnostic, and the biology can point in any direction. But the area that we are mainly focused on right now is the role of the microbiome, particularly with respect to human immunity. \\ If for whatever reason we decide that this is not a space that we want to continue to work in, though, the kinds of folks that we've hired are amazingly broad thinkers and we can pivot to another area of interesting and emerging science.}

At the same time, because Merck is a large integrated pharmaceutical company, we have enormous resources, capabilities and expertise that we can access throughout our organization. We are not meant to be a standalone discovery biology centre. Rather, our aim is to provide new insights and take advantage and utilize all of the amazing resources that exist within the broader Merck network.

\section{Q. Are you still on track to expand this exploratory R\&D effort?}

Our intent is to set up several centres, including this one in Cambridge. For many years now we've had a presence on the West Coast, but our new site in south San Francisco will be a very large integrated drug discovery centre that will not only consolidate all of the discovery work that has been going on on the West Coast, but that will also expand our footprint in the Bay Area.
Ageing and neuroscience will be a focus of the new research centre that's being set up in London.

Merck has always been committed to being a science-based company with a strong presence in basic discovery. The fact that we're setting up and expanding these centres I think really speaks to that commitment. It would be very sad if large companies were to exit the discovery space, because this is just the most exciting time to be in biological research.

\section{Q What are the low-hanging therapeutic opportunities in the microbiome, the focus} of your first Exploratory Science Center? Is there any such thing as low-hanging fruit in drug discovery anymore? Maybe there never has been, but I think the fruit has gotten less and less low-hanging over time. In any case, there are three main areas that we're interested in.

The first is how the microbiome relates to immune-oncology drugs, for which there's a lot of really compelling data out there from quite a number of groups. It's very exciting to think about how we might be able to either use the microbiome as a predictor of whether or not someone is likely to respond to an immune-oncology drug, or to think about ways to manipulate the microbiomes of non-responders or poor responders to make them more responsive (Nat. Rev. Drug Discov. 17, 153-155; 2018).

The second is to really understand how the microbiome contributes to vaccine responses. Our focus is on understanding what happens in the very, very young as the microbiome is evolving, as well as what happens in older populations as the microbiome changes as a function of age.

The third area is equally important, I think, but I'm not sure that there is as much emphasis on it now as there was many years ago. We are continuing to expand on observations that were made many years ago about how antibiotics impact the microbiome, and how this can contribute to pathogen defence or a lack thereof, as well as to response to vaccination. Merck is still really invested in all aspects of infectious disease research, and that includes the discovery and development of new antibiotics and antifungals. And by understanding that relationship, we might be able to do a better job of making the next generation of antibiotics and antifungals, which might have better outcomes if they have a lower probability of impacting the microbiome and pathogen defence.

\section{Q What would these next-generation antibiotics look like?}

In the absence of a true understanding of what's going on, it's difficult to say. It could mean narrower-spectrum antibiotics. Personally I don't think that's necessarily going to be the answer, although I wouldn't take it off the table. But if we can really understand which commensals are being affected and where those commensals reside, then maybe we can think about restricting access to antibiotics and developing drugs with pharmacokinetic properties and therapeutic windows that allow good activity against the pathogen while mitigating what's going on in terms of the rest of the microbiome.

It turns out that we're not sure if all antibiotics impact the overall microbiome, or only a subset of antibiotics, or if the duration of therapy matters. These are all very important things that we really need to try to tease out to be able to make better next-generation agents.

\section{Q In the broader context of microbiome opportunities, how do you think about live biotherapeutics versus small molecules? The live biotherapeutics obviously raise significant challenges, for example in}


terms of really understanding dose and dose-responses. And the bacteria that you're providing may have a benefit in one context but untoward effects in another context. It's a lot more challenging compared with the small-molecule approach. But I'm biased; I've been in small-molecule rational drug design for my entire career at Merck.

That said, I think the live biotherapeutics are probably the fastest way to get proof-of-concept data, at least in certain indications. The microbiome differs a lot from mice to primates to humans, and your microbiome is very different from my microbiome. But if you believe that important biology is conserved, really understanding the metabolites that are being made and the pathways that are being interdicted is where you're likely to see the most robust effects. And I think live biotherapeutics offer a really valuable tool for assessing that.

Q Your microbiome focus highlights in part Merck's continued interest in antibiotics, an area that most other large pharmaceutical firms have exited. How much antibiotic discovery and development work is Merck still doing?

We do quite a lot of work here. We have what I call traditional approaches, to see how we can improve upon known mechanisms of action. For example, we have an imipenemrelebactam combination that we hope will be approved soon. And we are working on treatment options for Gram-negative pathogens that carry drug resistance through the metallo- $\beta$-lactamase mechanism, to add to combination regimens. I would classify those as the more traditional programmes.

We also have very active discovery efforts looking for novel targets. That has been the holy grail of antibacterial discovery for several decades. Our focus in that area has shifted a bit, and while we're still interested in things that are broad spectrum, we've focused a little bit more in that area to think about pathogen-specific approaches that might be more tractable.

And then we are also working on things that are less traditional, such as antibody approaches for Gram-negative species that target the pathogen directly.
Q How problematic is the antibiotic business model for your continued work in this space? It is true that it is a problem that if we come up with something that is totally new and addresses drug resistance, its use will be reserved. And the return on that investment is not going to be there in the short term. But we believe it will be there in the longer term. And if different incentives are created, I'm sure it will make this space far more attractive not just for us but for a lot of other people as well.

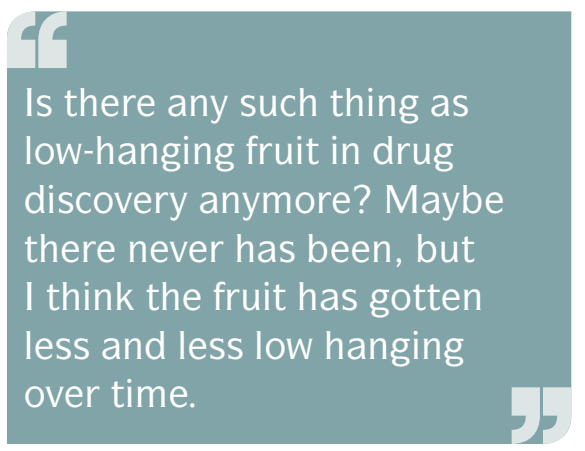

\section{Q. As vice president of infectious diseases and vaccine discovery, you also oversee Merck's HIV research, an area where you started your career. What are the next steps forward towards HIV cure?}

HIV cure is really part of what I call an eradication agenda. We really want to eradicate HIV, just like we want to eradicate hepatitis $\mathrm{C}$ and HPV. These are all infections where there's no animal reservoir, and so in principle if you eliminate the human reservoir you will eliminate the infection. That would be amazing.

But it's going to take a multipronged approach to effectively eradicate HIV. I think it's going to take either a vaccine or a really effective way to prevent transmission using antiretroviral drugs, which we know work really well if people take them. Because of the complexity of the populations who are affected and because of the complexity of the biology of HIV, multiple approaches are really going to be necessary. We have to find better ways to treat people, to get the medications to the people who are infected, and to find out who is really infected. And then we have to have more effective ways to deliver antiretroviral drugs to areas where HIV is endemic to prevent transmission. None of these things are trivial, and to get there we still need better agents.

It's not that the agents that we have aren't amazing, because they are. But you can always do better. Our phase II candidate MK-8591 fits into that category, because the compound is just so amazingly potent. It has such an incredible pharmacology that it can be dosed daily, monthly and maybe even formulated into longer-acting versions. If you have agents that have that sort of pharmacological forgiveness and offer the ability to dose people that infrequently, then you can start to really think seriously about getting antiretroviral therapy more broadly to the populations that are most affected. Because the currently available once-daily medications are challenging for people.

\section{Q Will you need to combine MK-8591 with other long-acting agents for efficacy?} It depends on whether you're speaking about prevention or treatment. For prevention, we have published some compelling animal data that show that MK- 8591 by itself works really well in preventing acquisition of infection. We think that as a single agent it will be very effective in prevention. But for treatment I do think we will need at least one other drug, and that is currently a very active ongoing effort that we are working on.

What about hopes for kick-and-kill
strategies, in which drugs are used first to
re-activate the hard-to-kill latent infected
cells and then to purge these from the body?
We're still in the very early days of truly
understanding the biology of these latent
cells. A large part of what we're doing
internally is developing tools that will help us
to really understand what's going on in HIV
reservoirs and why the virus recrudesces
after you take people off of antiretroviral
therapy. If you look in the plasma of HIV-
infected patients, it's very difficult to find
the latently infected cells. But if you look in
tissues you can see there's quite a bit of virus
and antigen being produced. And we need to
be better able to access those compartments
on an ongoing basis so that we can see what's
happening over time and with different
interventions. It's very challenging.

